\title{
Corrigendum: TET2 repression by androgen hormone regulates global hydroxymethylation status and prostate cancer progression
}

Ken-ichi Takayama, Aya Misawa, Takashi Suzuki, Kiyoshi Takagi, Yoshihide Hayashizaki, Tetsuya Fujimura, Yukio Homma, Satoru Takahashi, Tomohiko Urano \& Satoshi Inoue

Nature Communications 6:8219 doi: 10.1038/ncomms9219 (2015); Published 25 Sep 2015; Updated 14 Dec 2015

Previous work by Nickerson et al. reporting somatic TET2 mutations in metastatic castration-resistant prostate cancer was inadvertently omitted from the reference list of this Article and should have been cited at the end of the discussion section as reference 48 .

Nickerson, M. L. et al. Somatic alterations contributing to metastasis of a castration resistant prostate cancer. Hum. Mutat. 34, 1231-1241 (2013). 